\title{
STOKES PHENOMENA
}

\author{
BY YASUTAKA SIBUYA ${ }^{1}$
}

Communicated by H. F. Weinberger, April 18, 1977

1. Introduction. Let $x$ be a complex variable, and set $D(R)=\{x ;|x|>R\}$. Consider two differential equations

$$
d y / d x=A_{1}(x) y
$$

and

$$
d u / d x=A_{2}(x) u,
$$

where $y$ and $u$ are $n$-dimensional vectors, and $A_{1}$ and $A_{2}$ are $n$-by- $n$ matrices whose components are holomorphic in $D\left(R_{1}\right)$ and $D\left(R_{2}\right)$ respectively and meromorphic at $x=\infty$. Note that if $A_{1}$ and $A_{2}$ are replaced by their Laurent expansions at $x=\infty$, then $\left(E_{1}\right)$ and $\left(E_{2}\right)$ become two differential equations defined in a vector space over the field of quotients of the ring of formal power series in $x^{-1}$. The two equations $\left(E_{1}\right)$ and $\left(E_{2}\right)$ are said to be formally equivalent if there exists an $n$-by- $n$ matrix $T(x)$ such that (i) $T(x)=x^{q} \sum_{h=0}^{\infty} T_{h} x^{-h}$ is a formal series (with an integer $q$ and constant matrices $T_{h}$ ), (ii) det $T(x) \neq 0$ as a formal series, and (iii) the transformation $u=T(x) y$ reduces $\left(E_{1}\right)$ to $\left(E_{2}\right)$ formally. The two equations $\left(E_{1}\right)$ and $\left(E_{2}\right)$ are said to be meromorphically equivalent if there exists an $n$-by- $n$ matrix $T(x)$ such that (i) the components of $T(x)$ are holomorphic in $D\left(R_{3}\right)$ for some $R_{3}$ and meromorphic at $x=\infty$, (ii) det $T(x) \neq 0$ in $D\left(R_{3}\right)$, and (iii) the transformation $u=T(x) y$ reduces $\left(E_{1}\right)$ to $\left(E_{2}\right)$ in $D\left(R_{3}\right)$.

Let $\mathfrak{U}$ be an equivalence class of such differential equations with respect to the formal equivalence. The main concern in this report is to characterize the equivalence classes with respect to the meromorphic equivalence within $\mathfrak{Q}$.

2. Asymptotic properties of an analytic fibre bundle. For a sector $S$, set $S(R)=\{x \in S ;|x|>R\}$. An $n$-by- $n$ matrix $F(x)$ is said to have an asymptotic expansion $A_{s p}(F)$ as $x$ tends to $\infty$ in $S$, if (i) the components of $F(x)$ are holomorphic in $S(R)$ for some $R$, (ii) $A_{s p}(F)=x^{q} \sum_{h=0}^{\infty} G_{h} x^{-h}$ is a formal series, and (iii) for every positive integer $N$ there exists a positive number $K_{N}$ such that $\left|F(x)-x^{q} \sum_{h=0}^{N} G_{h} x^{-h}\right| \leqslant K_{N}|x|^{q-N-1}$ in $S(R)$. For an $n$-by- $n$ matrix $F(x)$, the notation $F(x) \in \mathrm{A}(S(R))$ means that (i) $F(x)$ is holomorphic

AMS (MOS) subject classifications (1970). Primary 34A20.

1 Supported by NSF MSC 76-06003. 
in $S(R)$, (ii) det $F(x) \neq 0$ in $S(R)$, and (iii) $F$ and $F^{-1}$ have asymptotic expansions $A_{s p}(F)$ and $A_{s p}\left(F^{-1}\right)$ respectively as $x$ tends to $\infty$ in $S$.

Throughout this section we fix a finite number of sectors $S_{1}, \ldots, S_{N}$. Set $S_{j}=\left\{x ; a_{j}<\arg x<b_{j}\right\}$. We assume that $0<b_{j}-a_{j}<\pi$ and that $\left\{S_{j}\right\}$ covers the entire $x$-plane. Set $S_{j k}=S_{j} \cap S_{k}$, where $(j, k)$ is an ordered pair. Let $\left\{F_{j k}(x)\right\}$ be a finite set of $n$-by- $n$ matrices such that (i) $F_{j k}(x) \in \mathrm{A}\left(S_{j k}(R)\right)$ for some $R$ whenever $S_{j k} \neq \varnothing$, and (ii) $F_{j h}(x) F_{h k}(x)=F_{j k}(x)$ whenever $S_{j} \cap$ $S_{h} \cap S_{k} \neq \varnothing$. Such a system $\left\{F_{j k}(x)\right\}$ is called an asymptotic system at $x=\infty$. An asymptotic system $\left\{F_{j k}(x)\right\}$ is said to be trivial at $x=\infty$, if there exist $n$ by- $n$ matrices $P_{1}(x), \ldots, P_{N}(x)$ such that (i) $P_{j}(x) \in \mathrm{A}\left(S_{j}(R)\right)$ for some $R$, and (ii) $F_{j k}(x)=P_{j}(x)^{-1} P_{k}(x)$ whenever $S_{j k} \neq \varnothing$. The set $\left\{P_{j}(x)\right\}$ is called a crosssection of $\left\{F_{j k}(x)\right\}$ at $x=\infty$. An asymptotic system $\left\{F_{j k}(x)\right\}$ is said to be formally trivial if there exist $n$-by- $n$ matrices $Q_{1}(x), \ldots, Q_{N}(x)$ such that (i) $Q_{j}(x)=x^{q_{j}} \sum_{h=0}^{\infty} Q_{j h} x^{-h}$ is a formal series, (ii) $\operatorname{det} Q_{j}(x) \neq 0$ as a formal series, and (iii) $A_{s p}\left(F_{j k}\right)=Q_{j}(x)^{-1} Q_{k}(x)$ whenever $S_{j k} \neq \varnothing$.

THEOREM 1. An asymptotic system $\left\{F_{j k}(x)\right\}$ is trivial at $x=\infty$ if and only if it is formally trivial.

To prove this theorem, the following lemma is essential.

LEMma 1. An asymptotic system $\left\{F_{j k}(x)\right\}$ is trivial at $x=\infty$ if $A_{s p}\left(F_{j k}\right)$ $=I$ (the identity matrix) whenever $S_{j k} \neq \varnothing$. (Cf. Y. Sibuya [3, Theorem 6.4.1, p. 172].)

THEOREM 2. Assume that an asymptotic system $\left\{F_{j k}(x)\right\}$ is trivial at $x=\infty$. Let $\left\{P_{j}(x)\right\}$ and $\left\{\widetilde{P}_{j}(x)\right\}$ be two cross-sections of $\left\{F_{j k}(x)\right\}$ at $x=\infty$. Then there exists an n-by-n matrix $T(x)$ such that (i) the components of $T(x)$ are holomorphic in $D(R)$ for some $R$ and meromorphic at $x=\infty$, and (ii) $\widetilde{P}_{j}(x)$ $=T(x) P_{j}(x)$ for every $j$.

3. Stokes structures. Let $\mathfrak{U}$ be an equivalence class of differential equations with respect to the formal equivalence (cf. §1). Let us fix an equation

$$
d y / d x=A_{0}(x) y
$$

which belongs to 2 . Let

$$
d u / d x=A(x) u
$$

be an arbitrary equation in 2 .

THEOREM 3. There exist a finite number of sectors $S_{1}, \ldots, S_{N}$ such shat (i) $\left\{S_{j}\right\}$ satisfies the two conditions given in $\S 2$, and (ii) for every equation $(E)$ in 2 there exist $n$-by-n matrices $P_{1}(x), \ldots, P_{N}(x)$ which satisfy the following conditions: (1) $P_{j}(x) \in A\left(S_{j}(R)\right)$ for some $R$, and (2) the transformation $u=P_{j}(x) y$ reduces $\left(E_{0}\right)$ to $(E)$ in $S_{j}(R)$. 
Throughout this section, we fix sectors $S_{1}, \ldots, S_{N}$ and fundamental matrix solutions $\Phi_{1}(x), \ldots, \Phi_{N}(x)$ of $\left(E_{0}\right)$ which are given in $S_{j}(R)$ for some $R$ (i.e. $d \Phi_{j}(x) / d x=A_{0}(x) \Phi_{j}(x)$ and $\operatorname{det} \Phi_{j}(x) \neq 0$ in $S_{j}(R)$ ) respectively.

A finite set $\left\{C_{j k}\right\}$ of $n$-by- $n$ constant matrices is called a Stokes system associated with थ2 if (i) $C_{j k} \in \mathrm{GL}(n, \mathbf{C})$ whenever $S_{j k} \neq \varnothing$, (ii) $C_{j h} C_{h k}=C_{j k}$ whenever $S_{j} \cap S_{h} \cap S_{k} \neq \varnothing$, (iii) $\Phi_{j}(x) C_{j k} \Phi_{k}(x)^{-1} \in A\left(S_{j k}(R)\right)$ for some $R$ whenever $S_{j k} \neq \varnothing$, and (iv) the asymptotic system $\left\{\Phi_{j}(x) C_{j k} \Phi_{k}(x)^{-1}\right\}$ is trivial at $x=\infty$. Two Stokes systems $\left\{C_{j k}\right\}$ and $\left\{\widetilde{C}_{j k}\right\}$ are said to be equivalent if there exist $n$-by- $n$ constant matrices $C_{1}, \ldots, C_{N}$ such that (i) $C_{j} \in \mathrm{GL}(n, \mathbf{C})$, (ii) $\Phi_{j}(x) C_{j} \Phi_{j}(x)^{-1} \in A\left(S_{j}(R)\right)$, and (iii) $C_{j k} C_{k}=C_{j} \widetilde{C}_{j k}$ whenever $S_{j k} \neq \varnothing$. An equivalence class $\mathcal{C}$ of Stokes systems is called $a$ Stokes structure associated with शr.

Let $\left\{P_{j}(x)\right\}$ be the matrices given in Theorem 3. Then $P_{j}(x) \Phi_{j}(x)$ is a fundamental matrix solution of $(E)$ in $S_{j}(R)$. Set $C_{j k}=\left(P_{j} \Phi_{j}\right)^{-1}\left(P_{k} \Phi_{k}\right)$ whenever $S_{j k} \neq \varnothing$. Then $\left\{C_{j k}\right\}$ is a Stokes system. Let $C$ be the Stokes structure to which $\left\{C_{j k}\right\}$ belongs. Then $C$ is independent of the choice of $\left\{P_{j}(x)\right\}$. This Stokes structure $C$ is called the Stokes structure determined by $(E)$ and denoted by $C(E)$.

THEOREM 4. For every Stokes structure $\mathcal{C}$ associated with $\mathfrak{A}$, there exists an equation $(E)$ in 2 such that $C=C(E)$.

THEOREM 5. Two equations $\left(E_{1}\right)$ and $\left(E_{2}\right)$ in 2 are meromorphically equivalent if and only if $C\left(E_{1}\right)=C\left(E_{2}\right)$.

REMARK. The definition of Stokes structures depends on the choice of $S_{j},\left(E_{0}\right), \Phi_{j}$. Another choice produces another isomorphic definition. Certain particular choices were given, for example, by G. D. Birkhoff [2] and Balser, Jurkat and Lutz [1].

\section{REFERENCES}

1. W. Balser, W. B. Jurkat and D. A. Lutz, Birkhoff invariants and Stokes' multipliers for meromorphic linear differential equations, Universitä̈ Ulm, 1976 (preprint).

2. G. D. Birkhoff, The generalized Riemann problem for linear differential equations and the allied problems for linear difference and q-difference equations, Proc. Amer. Acad. Arts and Sci. 49 (1913), 531-568.

3. Y. Sibuy a, Linear differential equations in the complex domain; problems of analytic continuation, Kinokuniya, Tokyo, 1976. (Japanese).

SCHOOL OF MATHEMATICS, UNIVERSITY OF MINNESOTA, MINNEAPOLIS, MINNESOTA 55455 